\title{
BIQ-BYWORD
}

Journal of Clinical and Nursing Research

\section{Application of Comfortable Nursing in the Preparation of Colostomy of Organs by Oral Magnesium Sulfate}

Zhou Shu

Department of Hematology, South Building, PLA General Hospital, Beijing 100853

\section{ARTICLE INFO}

Article history:

Published online: $30^{\text {th }}$ Sept, 2017

Key words:

Oral magnesium sulfate

Colonoscopy

Intestine preparation

Comfortable care

\begin{abstract}
Objective: To explore the application of comfortable nursing in the preparation of colonoscopy in oral magnesium sulfate, and to provide scientific reference direction for nursing staff. Methods: 68 patients with colonoscopy were randomly divided into experimental group and control group $(\mathrm{n}=34)$. In the control group, the routine nursing intervention was used, and the intervention group was treated with comfortable nursing intervention. Data analysis was performed using SPSS 20.0. Results: The total satisfaction score of the experimental group was $(96.88 \pm 1.26)$, the total satisfaction score of the control group was $(80.65 \pm 3.32)$, the test group $(\mathrm{P}<0.05)$. The incidence of adverse reactions in the experimental group was significantly lower than that in the control group. The incidence of adverse reactions was $5.88 \%$ in the experimental group and $23.53 \%$ in the control group. Conclusion: The effect of comfortable nursing on the preparation of colonic gut in the oral magnesium sulfate method is relatively large.
\end{abstract}

\section{Introduction}

With the change in people's diet, the incidence of intestinal disease increased significantly ${ }^{[1]}$. Electronic colonoscopy diagnosis and treatment is currently the most direct and effective way to understand intestinal diseases, but also the most reliable basis for the diagnosis of colonic lesions ${ }^{[2]}$. Traditional use of oral magnesium sulfate for a long time, this article is a new modified oral magnesium sulfate method, compared with the traditional method, the bowel preparation of the adverse reactions is small, increased patient satisfaction, and are reported as follows.

\section{Materials and methods}

\subsection{Information}

Subjects were selected from February 2015 to April 2016 in our hospital treated oral magnesium sulfate colonoscopy intestinal preparation of 68 patients, according to their admission order were randomly divided into test group and control group, each group of patients was for 34 cases. In the control group, the routine nursing intervention was used, and the intervention group was treated with comfortable nursing intervention.

\subsection{Methods}

\subsubsection{Intestinal preparation method}

By the nursing staff to explain to the patient in detail the preparation of the relevant methods of the intestine, $1 \mathrm{~d}$ before the inspection fasting, oral $50 \%$ magnesium sulfate $100 \mathrm{ml}$, drinking in $12 \mathrm{~min}, 30 \mathrm{~min}$ after a large number of drinking salt water, defecation after the electronic colonoscopy an examination.

\subsubsection{Nursing methods}

The control group used conventional nursing intervention methods, such as catering management and appropriate control of drinking water and other measures.

(1) Diet management: the patient before the inspection 
fasting treatment, so a small number of patients before the check dizziness and palpitation symptoms, nursing staff need to tell the patient $30 \mathrm{~min}$ before eating solid food and then check to improve the patient's resistance to colonoscopy acceptability

(2) Control the speed of drinking water: within $30 \mathrm{~min}$ divided into a small amount of magnesium sulfate solution to reduce the occurrence of vomiting, reduce the speed of drinking too fast and cause stomach swelling discomfort, improve the magnesium sulfate caused by electrolyte imbalance and other adverse reactions.

The experimental group on the basis of the implementation of the implementation of comfortable care methods, including the strengthening of psychological comfort care, comfortable environment care, medication comfort care.

(1) Psychological comfort care

because patients do not understand the colon endoscopy, and this inspection is an invasive operation, easy in the patient's psychological and physiological strong stress response, increase the patient's psychological Burden, aggravate the patient's discomfort, and even affect the smooth endoscopy, so the nursing staff in the patient before surgery should specify the specific steps and related precautions to reduce the patient' $\mathrm{s}$ anxiety and improve patient compliance.

(2) The environment comfortable care: to maintain the appropriate temperature and cleanliness of the ward, so that patients can feel the hospital's humanistic concept, to keep the toilet door lock in good condition to facilitate the use of patients.

(3) Medication comfort care: oral magnesium sulfate will appear vomiting and abdominal pain and other adverse reactions, nursing staff can guide the patient in the mouth when suffering from candy, bloating feeling when gently rub the abdomen; part of the weaker patients Should be closely observed changes in its condition, medication to the patient before the detailed notification of adverse reactions, severe diarrhea need to inform the doctor for related treatment promptly.

\subsection{Observe indicators}

The total satisfaction score (total score of 100 points), the incidence of adverse reactions and the degree of intestinal cleansing were analyzed. Intestinal cleaning: Class I for the whole colon without fecal and clear vision, will not affect the observation effect; II grade for the intestinal tract with a small amount of fecal and clear vision, and will not affect the observation effect; III level for the intestinal tract more Fecal and visual field blurred, affecting the intestinal wall observation effect; IV level for the intestinal wall full of feces, it is difficult to observe the effect of intestinal wall.

\subsection{Statistical methods}

SPSS 20.0 statistical software for data analysis, measurement data $( \pm \mathrm{s})$, the difference between groups, the difference between the use of $t$ test, count data comparison using $\times 2$ test, $\mathrm{P}<0.05$ was statistically significant.

\section{Results}

\subsection{Comparison of the two groups of patients with intestinal cleansing}

There were 20 cases of grade I, 10 cases of grade II, 3 cases of grade III and 1 grade of grade IV. Control group consisted of 19 cases of grade I, 11 cases of grade II, 2 cases of grade III and grade IV. There was no statistically significant difference in intestinal cleansing between the two groups $(\mathrm{P}>0.05)$

Table 1 Comparison of bowel cleansing in both groups $(x \pm \mathrm{s}, \mathrm{n})$

\begin{tabular}{ccccc}
\hline Item & Class I & Class II & Class III & Class IV \\
\hline Test group $(\mathrm{n}=34)$ & 20 & 10 & 3 & 1 \\
Control group $(\mathrm{n}=34)$ & 19 & 11 & 2 & 2 \\
$\mathrm{~T}$ & 12.3211 & 15.0121 & 16.2784 & 19.1023 \\
$\mathrm{P}$ & 0.1254 & 0.05621 & 0.0687 & 0.0712 \\
\hline
\end{tabular}

\subsection{The overall satisfaction score of the two groups of patients}

The overall satisfaction score of the test group was $(96.88$ $\pm 1.26)$ and the total satisfaction score of the control group was $(80.65 \pm 3.32)$. The total satisfaction score of the test group was much higher than that of the control group $(\mathrm{P}<0.05)$.

Table 2 Overall satisfaction score for both groups ( $x \pm \mathrm{s}$, points)

\begin{tabular}{cc}
\hline Item & Total Satisfaction Score \\
\hline Test group $(\mathrm{n}=34)$ & $96.88 \pm 1.26$ \\
Control group $(\mathrm{n}=34)$ & $80.65 \pm 3.32$ \\
$\mathrm{~T}$ & 10.2311 \\
$\mathrm{P}$ & 0.02113 \\
\hline
\end{tabular}




\subsection{Comparison of the incidence of adverse reactions in both groups}

The results showed that 1 case of nausea in the test group, 1 case of vomiting, 0 cases of abdominal pain, the total incidence of adverse reactions was $5.88 \%$; the control group 3 cases of nausea, 3 cases of vomiting, 2 cases of abdominal pain, the total incidence of adverse reactions was $23.53 \%$ The incidence of adverse reactions in the test group was significantly lower than that in the control group $(\mathrm{P}<0.05)$.

\section{Discussion}

Oral magnesium sulfate has always been an effective method for colonic endoscopic examination of intestine preparation. Magnesium sulfate for bowel preparation has the advantage that oral administration does not absorb hypertonic state in the intestine and can effectively reduce intestinal of the absorption, cause the intestinal volume gradually increased, causing diarrhea, patients do not need enema at this time, less pain ${ }^{[3,4]}$. Comfort care is a new concept and model of nursing in recent years. Through the study of the relationship between nursing activities and comfort, nursing staff can achieve an ideal state, or it can be said that the patient's physical and mental are in the most ideal of the state, to facilitate better treatment, so that patients can recover sooner ${ }^{[6-8]}$. The results of this study show that comfort care can better reflect the patient-centered service purposes so that patients can feel the hospital's intentions, and then greatly improve the overall satisfaction of patients with health care workers.

\section{Conclusion}

In summary, comfort care in the oral magnesium sulfate colonoscopy in the preparation of patients with colorectal play a greater role, can significantly improve the patient's overall satisfaction score, and improve the overall quality of care, reflecting the importance of comfort care value.

\section{References}

[1] Zhang Yuzhen, Wei Wanmei. Oral Administration of Magnesium Sulfate Colonoscopy in the Preparation of Nursing. Contemporary Nurses, 2014, 10(3): 91-93.

[2] Qin Juanwen. Colonoscopy before the Preparation of Intestinal Methods and Nursing Status. General Nursing, 2013, 11(11): 1031-1033.

[3] Lu Rui, Gao Daojian, Wang Shuping. Application Value of Magnesium Sulfate and Lactulose Mixed Solution in Intestine Preparation Before Colonoscopy. Chinese Journal of Digestive Endoscopy, 2014, 19(9): 513-514.

[4] Liao Ribin. Magnesium Sulfate Combined with Oral Rehydration Salt Colorectal Colonoscopy in The Treatment of Bowel Preparation Before Treatment. Chinese Journal of Misdiagnosis, 2010, 10(9): 2075-2076.

[5] Yao Liqin, Su Honglian, Xie Yuxia. Effect of Enteral Nutrition Solution on Bowel Preparation in Elderly Patients under Colonoscopy. Chinese Journal of Clinical Nursing, 2014, 15(3): 195-197.

[6] ZHENG Yu-ting, MIAO Xue-qin, MENG Xiaoxia. Effects of oral magnesium sulfate plus lotus seedlily meat decoction on intestinal preparation before electronic colonoscopy. Acta Nursing Journal, 2014, 21(23): 31-33.

[7] Luo Xiuhua, Feng Shuqin, Li Jie. Comfort Nursing in the Oral Magnesium Sulfate Intestine Preparation Colonoscopy in the Application. Practical Journal of Clinical Medicine, 2012, 16(14): 90-96.

[8] Zeng Xuyan, Cao Haijun, Li Shanao. The Effect of Magnesium Sulfate Combined with Phenolic Tablets and Oral Rehydration Salts on Intestinal Preparation. Chinese Journal of Digestive Endoscopy, 2013, 30(8): 464-465. 